\title{
Article
}

\section{Single-Shot Coherent X-Ray Imaging Instrument at PAL-XFEL}

\author{
Daeho Sung ${ }^{1,2,+}$, Daewoong Nam ${ }^{2,3,+} \oplus$, Myong-jin $\mathrm{Kim}^{3}$, Seonghan $\mathrm{Kim}^{3}$, Kyung Sook Kim ${ }^{2,3}$, \\ Sang-Youn Park ${ }^{2,3}{ }^{\circledR}$, Sun Min Hwang ${ }^{3}$, Chulho Jung ${ }^{1,2}$, Heemin Lee ${ }^{1,2}$, Do Hyung Cho ${ }^{1,2}$, Minseok Kim ${ }^{3}$, \\ Intae Eom ${ }^{2,3}$, Su Yong Lee ${ }^{4}$, Changyong Song ${ }^{1,2}$ and Sangsoo Kim ${ }^{3, *}$
}

1 Department of Physics, POSTECH, Pohang 37673, Korea; sdhsdh@postech.ac.kr (D.S.); jch2394@postech.ac.kr (C.J.); united4717@postech.ac.kr (H.L.); dohyung1230@postech.ac.kr (D.H.C.); cysong@postech.ac.kr (C.S.)

2 Photon Science Center, POSTECH, Pohang 37673, Korea; daewoong@postech.ac.kr (D.N.); kyungkim@postech.ac.kr (K.S.K.); sy.park@postech.ac.kr (S.-Y.P.); neplus@postech.ac.kr (I.E.)

3 XFEL Beamline Department, Pohang Accelerator Laboratory, Pohang 37673, Korea; mjk@postech.ac.kr (M.j.K.); kimsh80@postech.ac.kr (S.K.); hwangsm@postech.ac.kr (S.M.H.); kms83@postech.ac.kr (M.K.)

4 PLS-II Beamline Department, Pohang Accelerator Laboratory, Pohang 37673, Korea; dsleesy@postech.ac.kr

* Correspondence: sangsookim@postech.ac.kr

+ These authors contributed equally to the work.

check for updates

Citation: Sung, D.; Nam, D.; Kim, M.-j.; Kim, S.; Kim, K.S.; Park, S.-Y.; Hwang, S.M.; Jung, C.; Lee, H.; Cho, D.H.; et al. Single-Shot Coherent X-Ray Imaging Instrument at PAL-XFEL. Appl. Sci. 2021, 11, 5082 https://doi.org/10.3390/ app11115082

Academic Editors: Sooheyong Lee, Eric Landahl and Wojciech Roseker

Received: 2 May 2021

Accepted: 28 May 2021

Published: 30 May 2021

Publisher's Note: MDPI stays neutral with regard to jurisdictional claims in published maps and institutional affiliations.

Copyright: (c) 2021 by the authors. Licensee MDPI, Basel, Switzerland. This article is an open access article distributed under the terms and conditions of the Creative Commons Attribution (CC BY) license (https:/ / creativecommons.org/licenses/by/ $4.0 /)$.

\begin{abstract}
We developed a single-shot coherent X-ray imaging instrument at the hard X-ray beamline of the Pohang Accelerator Laboratory X-ray Free Electron Laser (PAL-XFEL). This experimental platform was established to conduct a variety of XFEL experiments, including coherent diffraction imaging (CDI), X-ray photon correlation spectroscopy (XPCS), and coherent X-ray scattering (CXS). Based on the forward-scattering geometry, this instrument utilizes a fixed-target method for sample delivery. It is well optimized for single-shot-based experiments in which one expects to observe the ultrafast phenomena of nanoparticles at picosecond temporal and nanometer spatial resolutions. In this paper, we introduce a single-shot coherent X-ray imaging instrument and report pump-probe coherent diffraction imaging (PPCDI) of Ag nanoparticles as an example of its applications.
\end{abstract}

Keywords: X-ray free electron laser; single-shot coherent X-ray imaging instrument; coherent diffraction imaging; pump-probe imaging; ultrafast dynamic imaging; ultrafast phenomena; temporal synchronization; fixed-target; thermal melting

\section{Introduction}

X-ray free electron lasers (XFELs) and diffraction-limited light sources are regarded as generating high-intensity and nearly coherent $X$-rays. Such coherent sources do not need a small aperture as a spatial filter to improve the spatial coherence. In contrast, synchrotron $\mathrm{X}$-ray sources are considered as generating relatively low-intensity and partially coherent $\mathrm{X}$-rays. They even lose most of their flux by using this small aperture to enhance the spatial coherence. Coherent diffraction imaging (CDI) of a single virus by synchrotron and XFEL sources is a good way in which to dramatically show the effect of X-ray sources $[1,2]$. Although both results revealed 2D structural information of viruses at nanoscale resolution, the data acquisition processes were quite different from each other. XFEL could easily achieve this feat with a single X-ray pulse, while a synchrotron source managed to do it with a large amount of data. Thus, high-intensity coherent XFEL enables us to conveniently perform a single-shot-based experiment. Furthermore, XFEL can be generated only in an ultrashort pulse form, which leads us to study ultrafast phenomena of nanoscience. Taking advantage of these remarkable properties of coherent light sources, CDI, X-ray photon correlation spectroscopy (XPCS), and other applications have been actively applied in various research fields [3-13].

We have developed a single-shot coherent $X$-ray imaging instrument to fully utilize the features of XFEL beams at the hard X-ray beamline of the Pohang Accelerator Laboratory 
X-ray Free Electron Laser (PAL-XFEL). This instrument was established to provide an optimum apparatus for single-shot-based X-ray experiments. In this paper, we present the details of the single-shot coherent X-ray imaging. Additionally, we briefly state the femtosecond laser system for optical pump X-ray probe experiments. Finally, we show the demonstration result of pump-probe coherent diffraction imaging (PPCDI) using this instrument. The melting process of Ag nanospheres is visualized at picosecond temporal and nanometer spatial resolutions.

\section{Materials and Methods}

After the first lasing of PAL-XFEL in 2016, the XFEL stability in the beam position and timing jitter were steadily improved for various X-ray experiments [14,15]. In 2017, a single-shot coherent $\mathrm{X}$-ray imaging instrument was successfully commissioned at the nano-crystallography and coherent imaging $(\mathrm{NCI})$ endstation at the hard X-ray beamline of PAL-XFEL. XPCS and CDI, which require highly coherent X-rays, were conducted using this instrument $[16,17]$.

\subsection{Overview of the Whole System}

The single-shot coherent $\mathrm{X}$-ray imaging instrument consists of a sample chamber for sample delivery, a loadlock chamber for fast sample exchange, and a detector part for photon measurement. The sample chamber is widely utilized with tightly focused X-ray pulses and optimized for single-shot-based experiments. Tightly focused X-ray pulses by Kirkpatrick-Baez mirrors (KB mirrors) with a $\sim 5.96 \mathrm{~m}$ long focal length are delivered to the sample chamber [18]. Since specimens are destroyed by a micron focused XFEL pulse [19], fresh samples are supplied to the interaction region using thin $\mathrm{Si}_{3} \mathrm{~N}_{4}$ membranes mounted on high-speed piezo stages. Diffraction patterns are collected by a multiport charge-coupled device (MPCCD) detector. To avoid damage to the detector by a direct beam, Ta metal is used as a beam stopper. Figure 1 shows the single-shot coherent $\mathrm{X}$-ray imaging instrument, including KB mirrors, at the NCI endstation of PAL-XFEL.

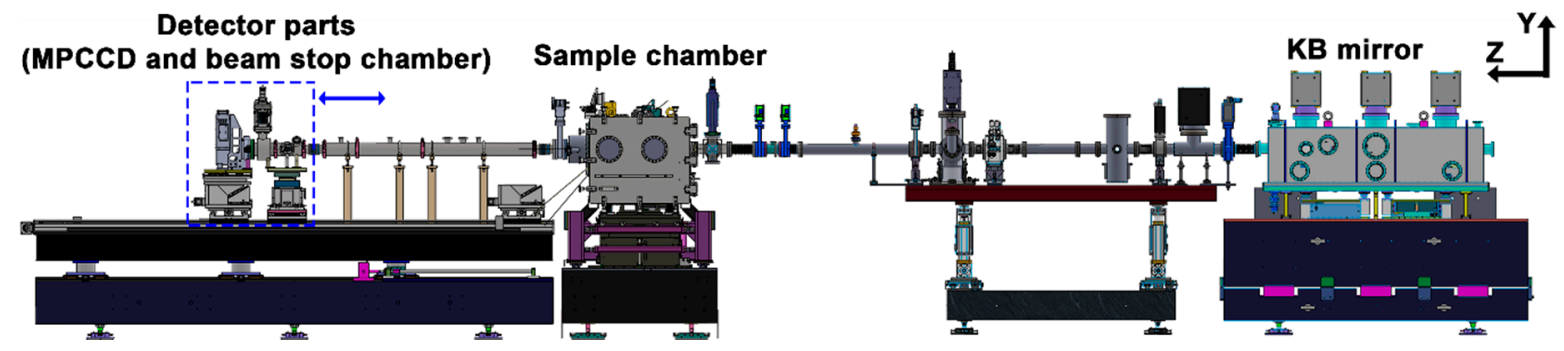

Figure 1. Single-shot coherent $\mathrm{X}$-ray imaging instrument at NCI endstation, Pohang Accelerator Laboratory $\mathrm{X}$-ray Free Electron Laser (PAL-XFEL). X-ray free electron laser (XFEL) pulses are focused by Kirkpatrick-Baez mirrors (KB mirrors) with a $\sim 5.96 \mathrm{~m}$ long focal length and delivered to the sample chamber. Fresh samples are supplied using a thin membrane to each X-ray pulse in the sample chamber. Diffracted signals from samples are collected by the multiport charge-coupled device (MPCCD). To avoid damage to the sensor, the beam stopper is located just upstream of the detector and blocks the direct X-ray beam. The detector parts marked by the blue box are mounted on the rail to easily change the distance between the sample and detector (the XFEL beam propagates following the z-axis in the figure).

\subsubsection{Sample Chamber and Loadlock Chamber}

Figure 2 shows a schematic view of the sample chamber. This chamber is optimized for single-shot-based XFEL experiments and designed to operate in vacuum and He gas ambient environments. It includes a beam position monitor, two four-jaw slits, a right-angle mirror, and a sample stage. As shown in Figure 2a, the sample chamber has five open ports: an incoming X-ray entrance port, a pump laser entrance port, and others for observing the interaction region. The port for the pump laser allows pump laser pulses to pass with an incident angle of 15 degrees with respect to the $\mathrm{X}$-ray. A clear aperture from this port to the $\mathrm{X}$-ray interaction region is secured to deliver the pump laser. The sample chamber stays 
on the XY-motion stage for its alignment to the XFEL beam. The chamber alignment can be confirmed through a beam position monitor that is equipped with a Ce:YAG (yttrium aluminum garnet) crystal and is located at its most upstream part. Downstream of the beam position monitor, two four-jaw slits block parasitic scattering mostly from the KB mirrors. Each slit blade is made of $250-\mu \mathrm{m}$-thick silicon with a 5-degree bevel cut and mounted on the linear stage to make a clean X-ray beam at the interaction region. Four motor stages are attached on the metal frame, which has a $5 \mathrm{~mm}$ by $5 \mathrm{~mm}$ clear square aperture for X-ray pass and a long hole on the right side for optical laser pass. The second slit is designed to place blades close to the interaction region using a cone (Figure 2b). A right-side blade has a 3-mm diameter hole for optical laser pass. The right-angle mirror with a 2-mm long hole is located between the second slit stage and the sample stage. The mirror is mounted on the $\mathrm{XY}$ linear motion stage to pass $\mathrm{X}$-ray pulses and optical laser pulses. To watch a membrane through a long-distance microscope (UWZ100, Union Optical Co., Ltd., Tokyo, Japan), this mirror is used. The working distance of the microscope is $100 \mathrm{~mm}$, with better than sub-10 $\mu \mathrm{m}$ image resolution. For fixed-target sample delivery, the membranes must be watched for X-ray alignment. As the X-ray interaction plane can be identified with the focal plane of the microscope, sample collection, beam size measurement, and timing synchronization are conducted at the same plane using the microscope. The sample stage consists of four high-precision motor stages. These motion stages are stacked as shown in Figure 3. A 200-mm long-stroke motion stage is piled at the bottom. As a coarse component, the motion stage is used to align each component. A hexapod stage was established in the middle of the sample stage. The hexapod serves all components placing the $\mathrm{X}$-ray interaction plane. Finally, two high-speed piezo stages are mounted on the hexapod. These are used to supply fresh samples to each X-ray pulse at the XFEL repetition rate of $60 \mathrm{~Hz}$. The maximum speed of these stages is $200 \mathrm{~mm} / \mathrm{s}$.
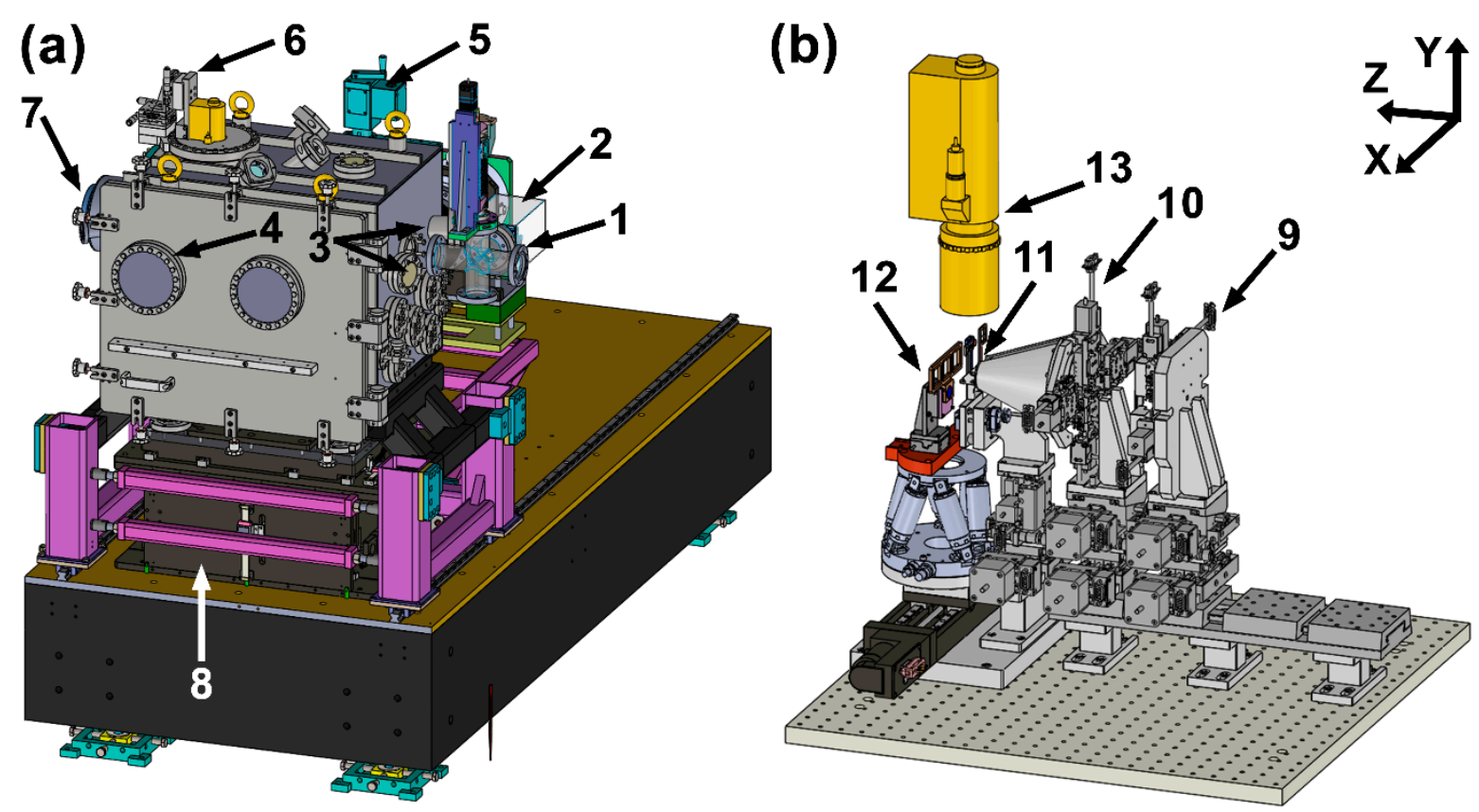

Figure 2. (a) The overall schematics of the sample chamber. (b) Internal components of the sample chamber. Major components are marked with numbers. (1) X-ray pulse entrance port. (2) Beam position monitor, Ce:YAG (yttrium aluminum garnet), with a linear stage. (3) Pump laser entrance ports with a fixed incident angle to the XFEL pulse at 15 degrees. (4) View port for the sample stage. (5) Gate valve. (6) Long working distance microscope with XYZ manual stages. (7) Exit port to the detector. (8) XY translation stage for the sample chamber. (9) Upstream four-jaw slit on the XY translation stage. (10) Downstream four-jaw slit with cone including XY translation stage. (11) Right-angle mirror with 2-mm clear long hole with XY translation stage and Z-axis manual stage. (12) Sample stage. (13) Long working distance microscope. 


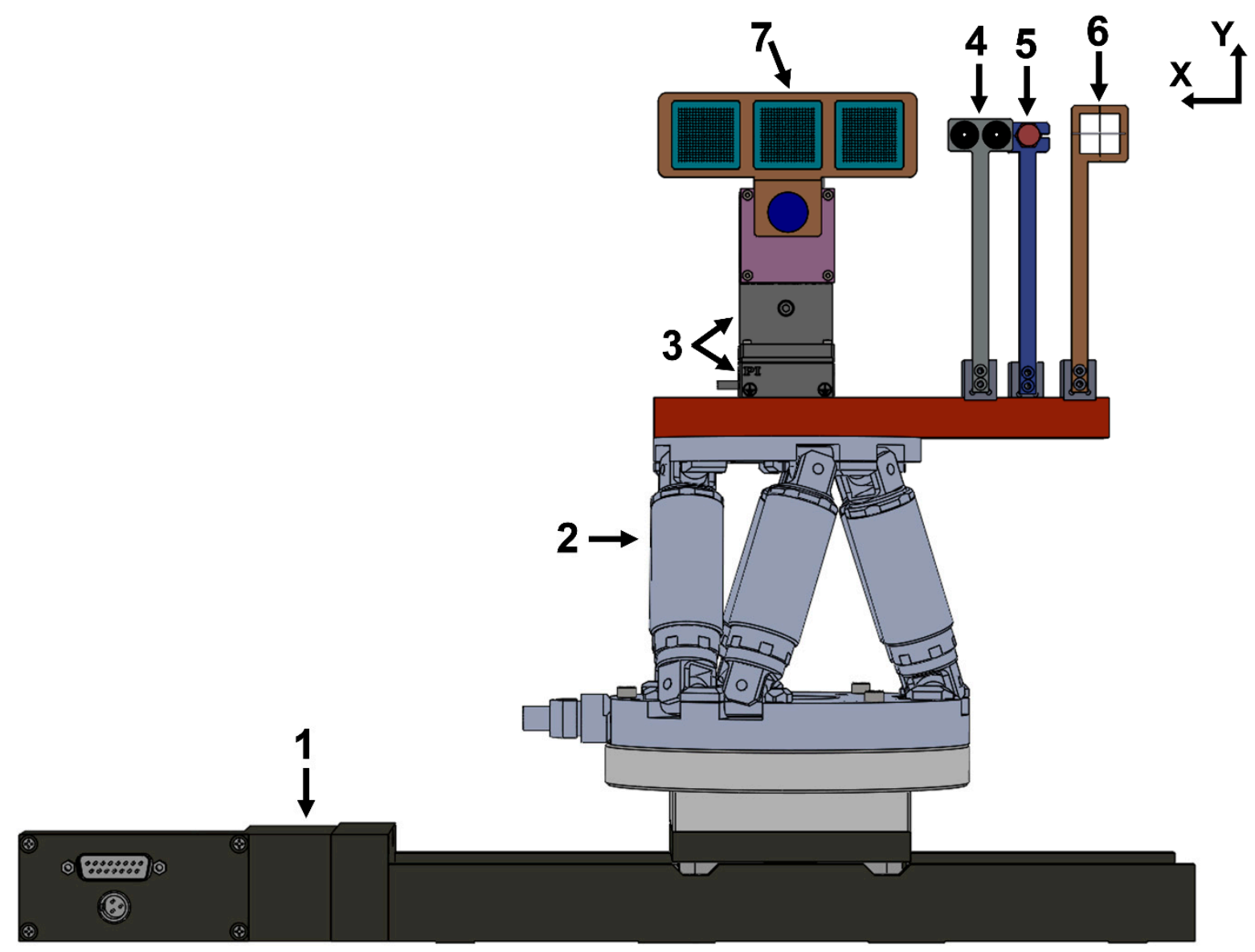

Figure 3. A schematic view of the sample stage with components marked by numbers. (1) Long stroke translation stage; (2) hexapod holding; (3) high-speed piezo XZ stages for raster scanning; (4) pinholes with $100 \mu \mathrm{m}$ diameter; (5) ultrafast photodetector for checking temporal overlap of pump and probe sources; and (6) 200- $\mu \mathrm{m}$ thick tungsten wire for measuring focused X-ray beam size. (7) Membrane holders and three membranes can be mounted at once.

The sample stage consisted of three $\mathrm{Si}_{3} \mathrm{~N}_{4}$ membranes for supplying fresh samples, a 200- $\mu \mathrm{m}$ thick tungsten wire for measuring the focused beam size by the knife-edge scan method, a pinhole with a diameter of $100 \mu \mathrm{m}$, and an ultrafast photodetector (G4176-03, Hamamatsu, Hamamatsu, Japan) for checking the spatial and temporal overlap between the pump and probe sources, respectively.

The loadlock chamber is attached to the sample chamber [20]. A key part of this loadlock system is the compact gripper. The gripper has two fingers, which can hold, release, and rotate a sample holder. These motions are activated by controlling pneumatic pressure. The sample holder contacting three $\mathrm{Si}_{3} \mathrm{~N}_{4}$ membranes can be mounted and removed using the loadlock system without venting the sample chamber.

\subsubsection{Sample Delivery Using a Thin $\mathrm{Si}_{3} \mathrm{~N}_{4}$ Membrane}

Fixed-target sample delivery using a thin membrane allows efficient provision of fresh samples with small sample consumption [21,22]. Using the high-speed property of the piezo stages and home-built scan program using Python, a raster scan is realized. A multirow membrane is employed instead of a multiwindow membrane. The multirow type has a more effective area, such as $\mathrm{Si}_{3} \mathrm{~N}_{4}$ only, and diminishes the risk of misalignment. When an X-ray pulse shoots the edge of the window by misalignment, strongly diffracted signals saturate the detector.

Each membrane has 2 by 36 windows with a $10 \mathrm{~mm}$ by $0.2 \mathrm{~mm} \mathrm{Si}{ }_{3} \mathrm{~N}_{4}$ area. One window is exposed to $\sim 600$ pulses with $50-\mu \mathrm{m}$ intervals between each shot. In total, $\sim 129,600$ diffraction patterns can be recorded by mounting three multirow membranes 
at once. The number of diffraction patterns collected by the raster scan depends on the interval between two spots by X-ray pulses. For PPCDI experiments, the interval is increased by $200 \mu \mathrm{m}$ because of the beam size of the optical laser. In this case, more than 10,000 diffraction patterns can be collected from three membranes.

\subsubsection{Detector Part}

The detector part contains the metal beam stopper with an XY manipulator, a photodiode, a Ce:YAG crystal, and the multiport charge-coupled device (MPCCD) detector, as shown in Figure 4. All components are mounted on the rails, and the sample-to-detector distance extends to $\sim 3.8 \mathrm{~m}$. The beam stop chamber is connected to the sample chamber through a vacuum beam path, and it is terminated by a Kapton film with $120 \mu \mathrm{m}$ thickness to separate the vacuum chamber and the MPCCD detector. The pressure of the whole system, the sample chamber, and the beam stop chamber is maintained in the vacuum range of $\sim 10^{-5}$ torr. The photodiode and the Ce:YAG crystal are mounted together by a linear manipulator. The photodiode is used to measure the focused beam size and check the flux of XFEL pulses. The position and shape of the XFEL beam are monitored by Ce:YAG crystal. The beam stopper is manipulated using an $X Y$ stage to block the direct beam. The beam stopper is made of 2-mm tantalum, and its edges are beveled at 5 degrees and polished. Alignment of the beam stopper is confirmed by a PD or a Ce:YAG crystal, which are mounted on the side of the MPCCD. The MPCCD is sealed by a $280-\mu$ m thick beryllium window and operates in a vacuum environment independently. This MPCCD consists of two sensors with a 300- $\mu \mathrm{m}$ overlapping area. Each sensor has 512 by 1024 pixels. The size of a pixel is $50 \mu \mathrm{m}^{2}$. The specification of the MPCCD has been reported elsewhere [23].

Recently, an adJUstiNg Gain detector FoR the Aramis User station (JUNGFRAU) 4 megapixel detector was readied for this XFEL experiment at PAL-XFEL. This JUNGFRAU detector has a $3.3 \mathrm{~mm}$ by $3.3 \mathrm{~mm}$ central hole to avoid damage by a direct $X$-ray beam. This detector can be connected to the sample chamber through a vacuum beam path without an air gap. It consists of eight sensors with $1.80 \mathrm{~mm}$ or $2.85 \mathrm{~mm}$ spacing. Each sensor has 512 by 1024 pixels. The size of a pixel is $75 \mu \mathrm{m}$ by $75 \mu \mathrm{m}$ [24].

\subsection{Pump Laser System}

Combining XFEL and a femtosecond pump laser enables us to carry out pump-probe experiments. As both sources generate femtosecond pulses, ultrafast dynamics in various fields can be studied $[9,24-27]$. The PAL-XFEL beamline is equipped with the femtosecond IR laser (Legend Elite DUO HE, Coherent Inc., Santa Clara, CA, USA) system to provide the pump source [28]. The maximum pulse energy of the fundamental mode $(\lambda=800 \mathrm{~nm})$ is $5 \mathrm{~mJ}$, and its pulse duration is $\sim 40 \mathrm{fs}$ in full width at half-maximum (FWHM) after passing a compressor. The harmonic generator $\left(\lambda_{2}=400 \mathrm{~nm}\right.$ and $\left.\lambda_{3}=266 \mathrm{~nm}\right)$ and the optical parametric amplifier are equipped to generate a broad wavelength range, covering from $240 \mathrm{~nm}$ to $2600 \mathrm{~nm}$. By reducing or extending the optical path using a motion stage, a time delay is changed for time-resolved experiments. Furthermore, a phase shifter will be established to change the time delay with improved beam pointing stability.

\section{Timing Synchronization}

It is essential to define the time zero between two light sources, $\mathrm{X}$-rays and optical lasers, with femtosecond precision to observe ultrafast phenomena. It realizes high precision time synchronization by detecting the transmitted intensity of the optical laser, which passes through a Ce:YAG crystal. When the Ce:YAG crystal is illuminated by X-rays first, the transmission rate of the optical laser is lower than its native state. By capturing the change in the transmission rate as a time series function, timing synchronization with femtosecond precision can be defined $[29,30]$.

We performed timing synchronization using the Ce:YAG crystal. First, coarse temporal overlap with a resolution of a few tens of picoseconds was set using an ultrafast photodetector. As a probe source, the incident X-ray energy was set at $5 \mathrm{keV}(0.248 \mathrm{~nm}$ 
wavelength) with a $\sim 20-\mathrm{eV}$ bandwidth. The transmission of the femtosecond IR laser was measured while changing the time delay. The change in transmitted intensity was clearly observed without jitter correction, as shown in Figure 5. At a positive delay, the intensity of the transmitted IR laser remains constant. The transmission intensity declines at a negative delay since X-ray pulses arrive first and cause the metallization process of the Ce:YAG crystal. We observed the transient transmission changes of the IR laser pulses and set the time zero with sub-picosecond temporal resolution.

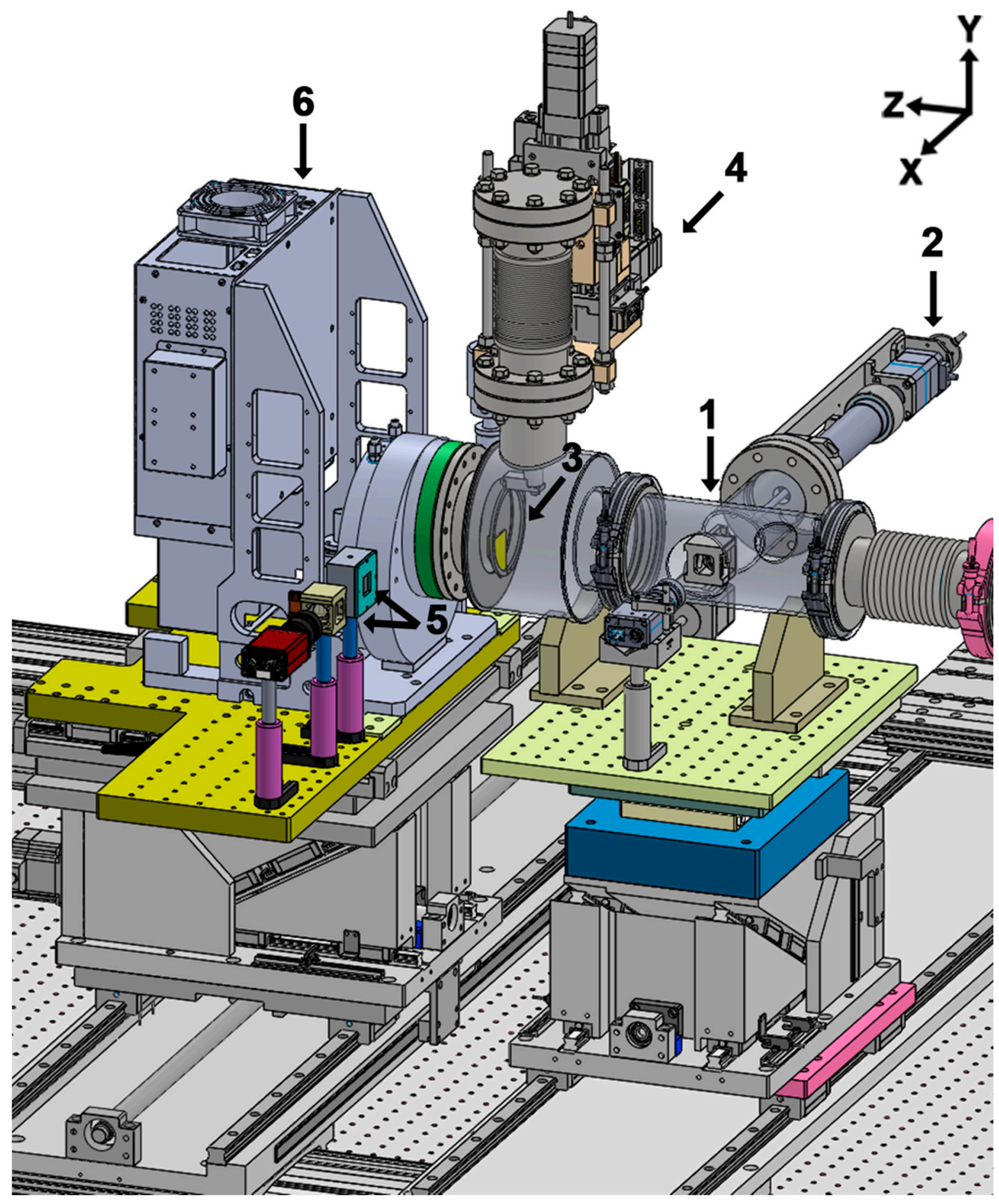

Figure 4. A schematic view of the detector part. (1) Beam position monitor, photodiode, and YAG crystal. (2) Linear manipulator for beam position monitor. (3) Ta metal beam stopper with $2 \mathrm{~mm}$ thickness. (4) XY manipulator for the beam stopper. (5) Beam position monitor, photodiode, and YAG crystal. (6) MPCCD detector. 


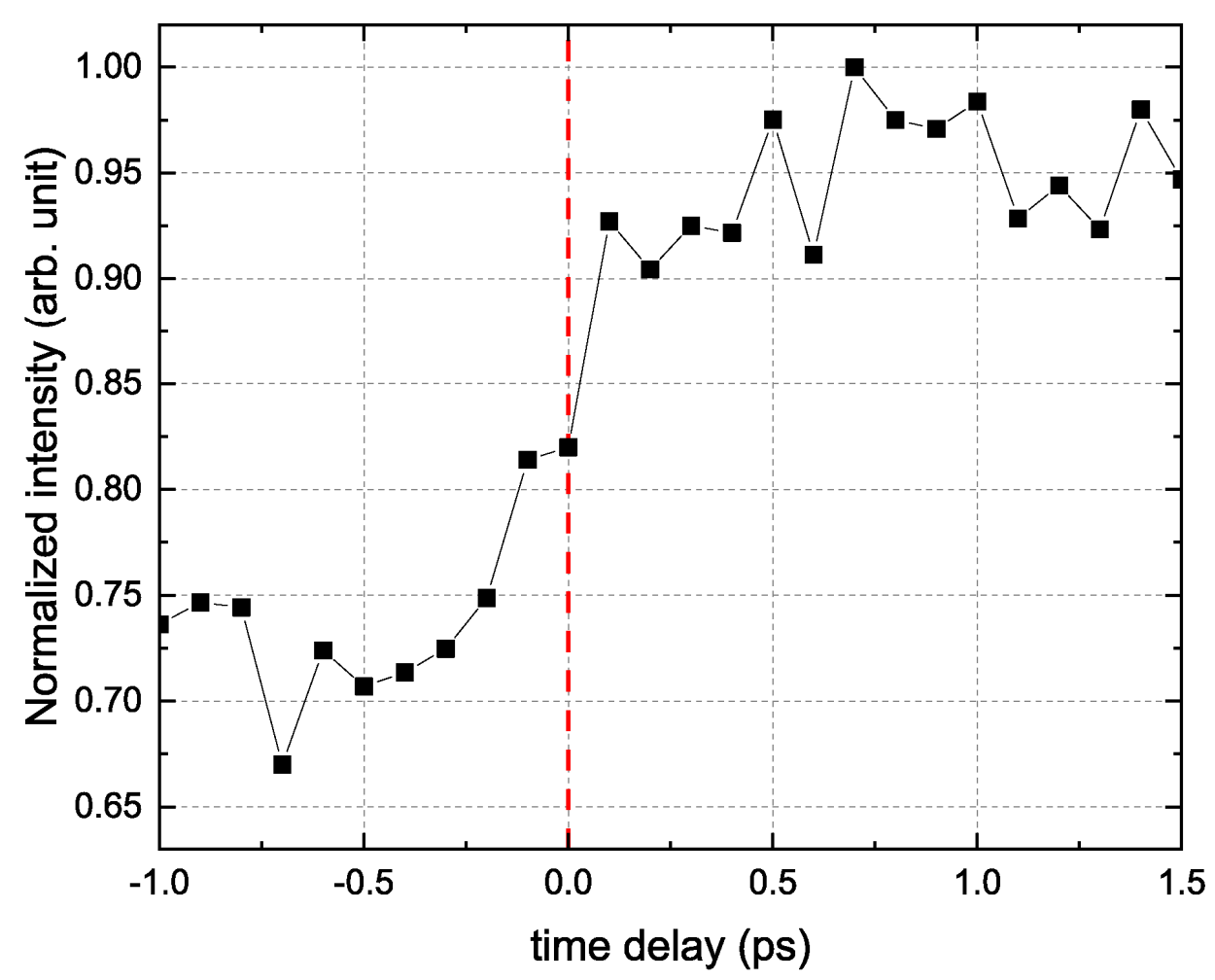

Figure 5. Timing synchronization of two light sources with femtosecond precision using a Ce:YAG. The intensity of the transmitted IR laser is kept constant at negative delays. The intensity is reduced when X-ray pulses arrive first.

\section{Results}

We conducted a single-shot PPCDI experiment to observe melting process of Ag nanosphere. The experiment was performed at the NCI endstation in the hard X-ray beamline of PAL-XFEL. It was designed to show a typical application of the developed instrument. A femtosecond IR laser with a 100-fs pulse duration was used as a pump source. The pump laser with $180 \mu \mathrm{m} \times 200 \mu \mathrm{m}$ size (FWHM) was illuminated on the sample plane. The laser fluence was $\sim 420 \mathrm{~mJ} / \mathrm{cm}^{2}$, which can increase sample temperature up to $1290 \mathrm{~K}$ [31]. As the melting temperature of $\mathrm{Ag}$ is $1235 \mathrm{~K}$, this fluence is enough to cause thermal melting of Ag nanoparticles.

The X-ray energy was fixed to $5 \mathrm{keV}$, which corresponds to $0.248 \mathrm{~nm}$ in wavelength. A $\mathrm{KB}$ mirror was used to deliver a focused beam with $7 \mu \mathrm{m} \times 9 \mu \mathrm{m}$ size (FWHM). Highly monodisperse Ag nanospheres with $100 \mathrm{~nm}$ diameter (nanoComposix, San Diego, CA, USA) were prepared for the sample. Before dispersing nanospheres on the $\mathrm{Si}_{3} \mathrm{~N}_{4}$ membrane, the membrane surface was charged to increase the wettability by using a plasma cleaner. Ag nanospheres were ultra-sonicated to avoid the aggregation. Additionally, a spin coater was used to promote uniform dispersion on the membrane [22]. Fresh specimens were provided to each X-ray pulse by using high-speed piezo stages. Diffraction patterns were collected through the MPCCD detector. The detector was placed $1.5 \mathrm{~m}$ downstream of the sample.

Diffraction patterns were classified according to time delay, hit or nonhit status, and single or multiple particles. Figure $6 \mathrm{a}, \mathrm{c}, \mathrm{d}$ show three representative diffraction patterns from single Ag nanoparticles acquired at different time delays: 0 ps, 16 ps, and 45 ps. For phase retrieval, a generalized proximal smoothing (GPS) algorithm was used [32]. The reconstructed images are shown in the insets of Figure 6a,c. A diffraction pattern, which is similar to the airy rings, reflects the shape and the size of the specimen. In the far field, the amplitude of the diffraction pattern is the Fourier transform of the electron density 
distribution of the sample. The samples to be measured are Ag nanospheres of uniform density, the diffraction amplitude $\mathrm{F}(\mathrm{s})$ is as follows.

$$
\mathrm{F}(\mathrm{s})=\frac{4}{3} \pi \mathrm{R}^{3} \Phi(2 \pi \mathrm{s}),
$$

where, $\Phi(\mathrm{u})=3 \frac{(\sin \mathrm{u}-\mathrm{u} \cos \mathrm{u})}{\mathrm{u}^{3}}$.
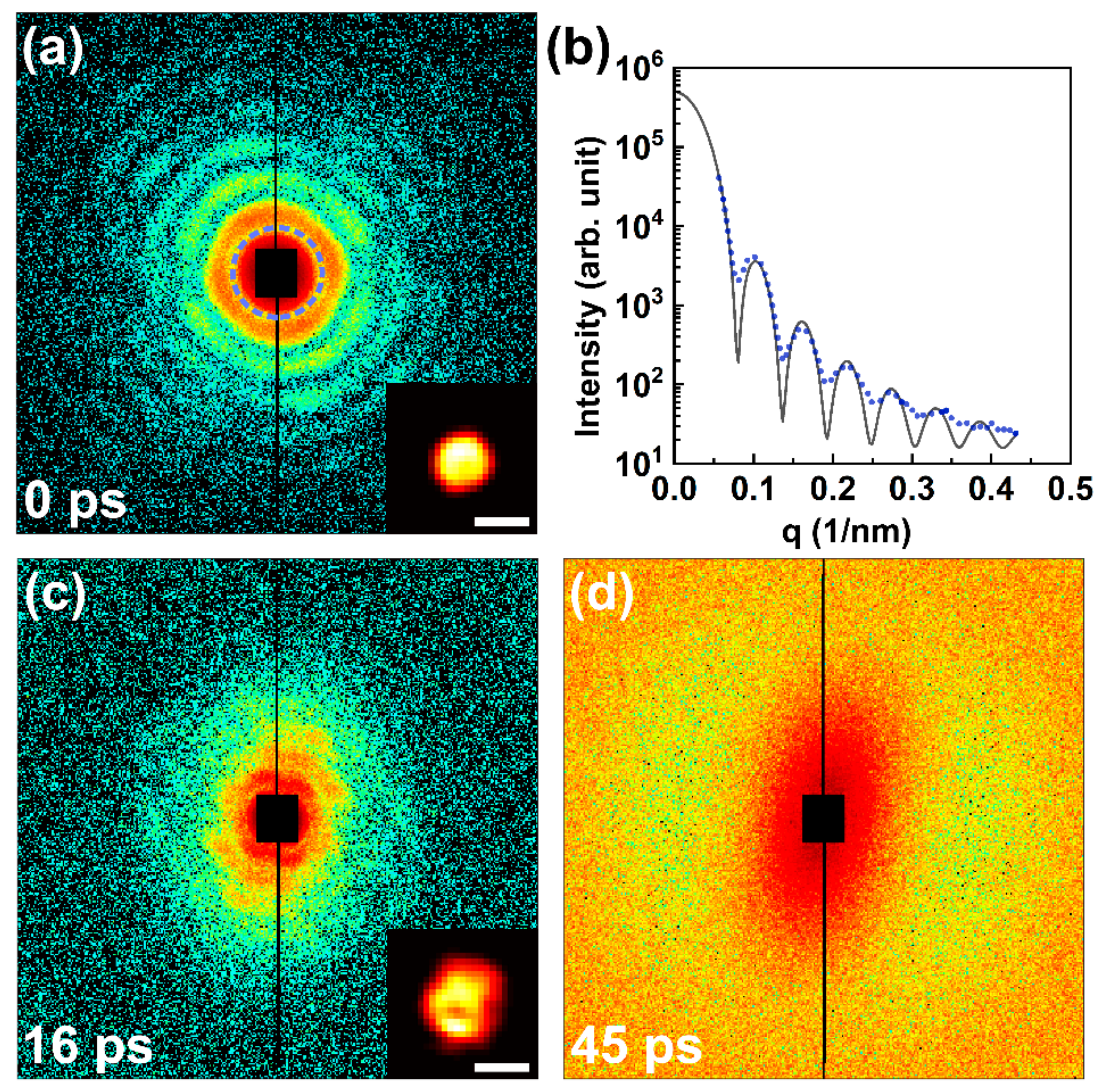

Figure 6. Diffraction patterns measured over a series of time delays showing the Ag nanoparticle melting process. Ag nanoparticles with a nominal diameter of $100 \mathrm{~nm}$ were melted by the IR laser ( $\lambda=800 \mathrm{~nm}, \sim 420 \mathrm{~mJ} / \mathrm{cm}^{2}$ fluence). The intact Ag nanosphere reconstructed from diffraction pattern measured at 0 ps time delay is shown in the inset of (a). The blue dashed circle of the diffraction pattern in (a) represents the size of the zeroth speckle. The $q_{\text {zeroth }}$ corresponding to the radius of this circle is $0.08 \mathrm{~nm}^{-1}$. (b) The sum of the radial intensity (blue dotted line) for the diffraction pattern of the intact Ag nanosphere is fitted to the sum of the radial intensity of the ideal sphere of the same size expected from Equation (1) (black line). (c) The measured diffraction pattern after a time delay of 16 ps shows the morphological transformation and expansion of the nanoparticles by the IR laser. The low-density void is shown in the reconstructed image (the inset of (c)). The butterfly-shaped signal was measured after a 45-ps time delay (d). As there is no speckle at all, this signal indicates the complete destruction of the nanosphere. The length of scalebar corresponds to $100 \mathrm{~nm}$.

Here, $\mathrm{R}$ is the radius of the sphere, and Equation (1) is the Fourier transform of a uniform sphere. The intensity of the measured diffraction pattern can be estimated from the square of Equation (1).

Figure 6a shows the diffraction pattern measured on an intact Ag nanosphere. The blue dashed circle in Figure 6a represents the momentum transfer of the zeroth speckle, $\mathrm{q}_{\mathrm{zeroth}}$, and its radius is $0.08 \mathrm{~nm}^{-1}$. The diameter of the actually measured Ag nanosphere can be estimated by Equation (1), and its value is $\sim 112 \mathrm{~nm}$. In addition, the radial intensity sum of the diffraction pattern in Figure $6 a$ is fitted to the radial intensity sum of the 
same diameter ideal sphere calculated by Equation (1) (Figure 6b). This indicates that the measured sample is close to the ideal sphere of $\sim 112 \mathrm{~nm}$ diameter. The reconstructed image in the inset of Figure 6a intuitively displays the shape of the sample. The diffraction pattern in Figure $6 \mathrm{c}$ shows the shrinkage of the speckles size and the reduction of visibility at the 16-ps time delay. This diffraction pattern is a snapshot of the melting process of the Ag nanosphere. Heat transfer from electrons to the lattice excited by a femtosecond infrared laser leads to the melting and thermal expansion of the Ag nanosphere. The narrower pattern width, compared to the diffraction pattern in Figure 6a, is the result of the thermal expansion of the Ag nanosphere. The distorted diffraction pattern in Figure $6 \mathrm{c}$ implies a deformation of the shape of the $\mathrm{Ag}$ nanosphere during the melting process, which is directly observable in the reconstructed image (the inset of Figure 6c). In the reconstructed image in the inset of Figure 6c, the sample deformed and expanded during melting. At the same time, a low-density void formed in the center. After the 45-ps time delay, an anisotropic butterfly-shaped diffraction signal appeared (Figure 6d). As there was no observable speckle, it can be inferred that the Ag nanosphere was completely destructed. The process of the Ag nanoparticle melting is similar to the previous result [9].

\section{Discussion}

We introduced a single-shot coherent $\mathrm{X}$-ray imaging instrument, which can be applied in various coherent $X$-ray experiments, at the NCI endstation of PAL-XFEL. This instrument was well optimized for single-shot-based XFEL experiments, including pump-probe experiments. To supply fresh samples, raster scanning was enabled using high-speed piezo stages and home-built software.

Additionally, we demonstrated a single-shot PPCDI in which we observed the ultrafast melting phenomena of Ag nanoparticles. The result shows that single-shot PPCDI can visualize the ultrafast phenomena of nanoparticles at picosecond temporal and nanometer spatial resolutions. In particular, it can be applied to understand irreversible phenomena in the case of homogenous specimens.

Now, we are continuing to upgrade the single-shot coherent $\mathrm{X}$-ray imaging instrument. The JUNGFRAU detector has been ready for single-shot XFEL experiments. It has high detection efficiency from tender X-rays to over $10 \mathrm{keV}$ [33]. To achieve 3D single-particle imaging, we are developing a gas jet injector for the sample delivery system. To enhance the flux density of XFEL dramatically, we plan to install nanofocusing optics in the near future.

Author Contributions: Conceptualization: S.K. (Sangsoo Kim) and C.S.; software: S.K. (Sangsoo Kim); formal analysis: D.S. and D.N.; investigation: all; writing-original draft preparation: D.S. and D.N.; writing-review and editing: all; visualization: D.S., D.N., M.K., and S.K. (Seonghan Kim); supervision: S.K. (Sangsoo Kim) and C.S. All authors have read and agreed to the published version of the manuscript.

Funding: This research was funded by the National Research Foundation of Korea, NRF-2018R1D1A1 B07048306 and NRF-2019R1A6B2A02098631, NRF-2019R1A2B5B03070059, and the Ministry of Science and ICT of Korea.

Institutional Review Board Statement: Not applicable.

Informed Consent Statement: Not applicable.

Data Availability Statement: The data presented in the study are available on request from the corresponding author.

Acknowledgments: We acknowledge the PAL-XFEL engineering team and accelerator division for enormous technical assistance to establish this instrument. The XFEL experiments were conducted at the hard X-ray beamline of PAL-XFEL.

Conflicts of Interest: The authors declare no conflict of interest. 


\section{References}

1. Song, C.; Jiang, H.; Mancuso, A.; Amirbekian, B.; Peng, L.; Sun, R.; Shah, S.S.; Zhou, Z.H.; Ishikawa, T.; Miao, J. Quantitative imaging of single, unstained viruses with coherent X-rays. Phys. Rev. Lett. 2008, 101, 158101. [CrossRef] [PubMed]

2. Seibert, M.M.; Ekeberg, T.; Maia, F.R.; Svenda, M.; Andreasson, J.; Jönsson, O.; Odić, D.; Iwan, B.; Rocker, A.; Westphal, D. Single mimivirus particles intercepted and imaged with an X-ray laser. Nature 2011, 470, 78-81. [CrossRef] [PubMed]

3. Bostedt, C.; Eremina, E.; Rupp, D.; Adolph, M.; Thomas, H.; Hoener, M.; de Castro, A.R.B.; Tiggesbäumker, J.; Meiwes-Broer, K.H.; Laarmann, T.; et al. Ultrafast X-ray Scattering of Xenon Nanoparticles: Imaging Transient States of Matter. Phys. Rev. Lett. 2012, 108, 093401. [CrossRef] [PubMed]

4. Clark, J.N.; Beitra, L.; Xiong, G.; Higginbotham, A.; Fritz, D.M.; Lemke, H.T.; Zhu, D.; Chollet, M.; Williams, G.J.; Messerschmidt, M.; et al. Ultrafast Three-Dimensional Imaging of Lattice Dynamics in Individual Gold Nanocrystals. Science 2013, 341, 56. [CrossRef] [PubMed]

5. $\quad$ van der Schot, G.; Svenda, M.; Maia, F.R.N.C.; Hantke, M.; DePonte, D.P.; Seibert, M.M.; Aquila, A.; Schulz, J.; Kirian, R.; Liang, M.; et al. Imaging single cells in a beam of live cyanobacteria with an X-ray laser. Nat. Commun. 2015, 6, 5704. [CrossRef]

6. $\quad$ Ekeberg, T.; Svenda, M.; Abergel, C.; Maia, F.R.N.C.; Seltzer, V.; Claverie, J.-M.; Hantke, M.; Jönsson, O.; Nettelblad, C.; van der Schot, G.; et al. Three-Dimensional Reconstruction of the Giant Mimivirus Particle with an X-ray Free-Electron Laser. Phys. Rev. Lett. 2015, 114, 098102. [CrossRef]

7. Hosseinizadeh, A.; Mashayekhi, G.; Copperman, J.; Schwander, P.; Dashti, A.; Sepehr, R.; Fung, R.; Schmidt, M.; Yoon, C.H.; Hogue, B.G.; et al. Conformational landscape of a virus by single-particle X-ray scattering. Nat. Methods 2017, 14, 877-881. [CrossRef]

8. Gorkhover, T.; Ulmer, A.; Ferguson, K.; Bucher, M.; Maia, F.R.N.C.; Bielecki, J.; Ekeberg, T.; Hantke, M.F.; Daurer, B.J.; Nettelblad, C.; et al. Femtosecond X-ray Fourier holography imaging of free-flying nanoparticles. Nat. Photonics 2018, 12, 150-153. [CrossRef]

9. Ihm, Y.; Cho, D.H.; Sung, D.; Nam, D.; Jung, C.; Sato, T.; Kim, S.; Park, J.; Kim, S.; Gallagher-Jones, M.; et al. Direct observation of picosecond melting and disintegration of metallic nanoparticles. Nat. Commun. 2019, 10, 2411. [CrossRef]

10. Lehmkühler, F.; Dallari, F.; Jain, A.; Sikorski, M.; Möller, J.; Frenzel, L.; Lokteva, I.; Mills, G.; Walther, M.; Sinn, H.; et al. Emergence of anomalous dynamics in soft matter probed at the European XFEL. Proc. Natl. Acad. Sci. USA 2020, 117, 24110. [CrossRef]

11. Roseker, W.; Lee, S.; Walther, M.; Lehmkühler, F.; Hankiewicz, B.; Rysov, R.; Hruszkewycz, S.O.; Stephenson, G.B.; Sutton, M.; Fuoss, P.H.; et al. Double-pulse speckle contrast correlations with near Fourier transform limited free-electron laser light using hard X-ray split-and-delay. Sci. Rep. 2020, 10, 5054. [CrossRef] [PubMed]

12. Lehmkühler, F.; Hankiewicz, B.; Schroer, M.A.; Müller, L.; Ruta, B.; Sheyfer, D.; Sprung, M.; Tono, K.; Katayama, T.; Yabashi, M.; et al. Slowing down of dynamics and orientational order preceding crystallization in hard-sphere systems. Sci. Adv. 2020, 6, eabc5916. [CrossRef] [PubMed]

13. Büttner, F.; Pfau, B.; Böttcher, M.; Schneider, M.; Mercurio, G.; Günther, C.M.; Hessing, P.; Klose, C.; Wittmann, A.; Gerlinger, K.; et al. Observation of fluctuation-mediated picosecond nucleation of a topological phase. Nat. Mater. 2021, 20, 30-37. [CrossRef] [PubMed]

14. Kang, H.-S.; Min, C.-K.; Heo, H.; Kim, C.; Yang, H.; Kim, G.; Nam, I.; Baek, S.Y.; Choi, H.-J.; Mun, G.; et al. Hard X-ray free-electron laser with femtosecond-scale timing jitter. Nat. Photonics 2017, 11, 708-713. [CrossRef]

15. Nam, I.; Min, C.-K.; Oh, B.; Kim, G.; Na, D.; Suh, Y.J.; Yang, H.; Cho, M.H.; Kim, C.; Kim, M.-J.; et al. High-brightness self-seeded X-ray free-electron laser covering the $3.5 \mathrm{keV}$ to $14.6 \mathrm{keV}$ range. Nat. Photonics 2021, 15, 435-441.

16. Yun, K.; Kim, S.; Kim, D.; Chung, M.; Jo, W.; Hwang, H.; Nam, D.; Kim, S.; Kim, J.; Park, S.-Y.; et al. Coherence and pulse duration characterization of the PAL-XFEL in the hard X-ray regime. Sci. Rep. 2019, 9, 3300. [CrossRef]

17. Cho, D.H.; Shen, Z.; Ihm, Y.; Wi, D.H.; Jung, C.; Nam, D.; Kim, S.; Park, S.-Y.; Kim, K.S.; Sung, D.; et al. High-Throughput 3D Ensemble Characterization of Individual Core-Shell Nanoparticles with X-ray Free Electron Laser Single-Particle Imaging. ACS Nano 2021, 15, 4066-4676. [CrossRef]

18. Kim, J.; Kim, H.-Y.; Park, J.; Kim, S.; Kim, S.; Rah, S.; Lim, J.; Nam, K.H. Focusing X-ray free-electron laser pulses using Kirkpatrick-Baez mirrors at the NCI hutch of the PAL-XFEL. J. Synchrotron Radiat. 2018, 25, 289-292. [CrossRef]

19. Barty, A.; Caleman, C.; Aquila, A.; Timneanu, N.; Lomb, L.; White, T.A.; Andreasson, J.; Arnlund, D.; Bajt, S.; Barends, T.R.M.; et al. Self-terminating diffraction gates femtosecond X-ray nanocrystallography measurements. Nat. Photonics 2012, 6, 35-40. [CrossRef] [PubMed]

20. Nam, D.; Kim, S.; Kim, M.-J.; Kim, S. Development of a Fast Sample-exchange System for Fixed Target Single-shot Imaging at PAL-XFEL. J. Korean Phys. Soc. 2020, 76, 448-452. [CrossRef]

21. Hunter, M.S.; Segelke, B.; Messerschmidt, M.; Williams, G.J.; Zatsepin, N.A.; Barty, A.; Benner, W.H.; Carlson, D.B.; Coleman, M.; Graf, A.; et al. Fixed-target protein serial microcrystallography with an X-ray free electron laser. Sci. Rep. 2014, 4, 6026. [CrossRef]

22. Nam, D.; Kim, C.; Kim, Y.; Ebisu, T.; Gallagher-Jones, M.; Park, J.; Kim, S.; Kim, S.; Tono, K.; Noh, D.Y.; et al. Fixed target single-shot imaging of nanostructures using thin solid membranes at SACLA. J. Phys. B Atom. Mol. Phys. 2016, 49, 034008. [CrossRef]

23. Kameshima, T.; Ono, S.; Kudo, T.; Ozaki, K.; Kirihara, Y.; Kobayashi, K.; Inubushi, Y.; Yabashi, M.; Horigome, T.; Holland, A.; et al. Development of an X-ray pixel detector with multi-port charge-coupled device for X-ray free-electron laser experiments. Rev. Sci. Instrum. 2014, 85, 033110. [CrossRef] 
24. Clark, J.N.; Beitra, L.; Xiong, G.; Fritz, D.M.; Lemke, H.T.; Zhu, D.; Chollet, M.; Williams, G.J.; Messerschmidt, M.M.; Abbey, B.; et al. Imaging transient melting of a nanocrystal using an X-ray laser. Proc. Natl. Acad. Sci. USA 2015, $112,7444$. [CrossRef] [PubMed]

25. Orville, A.M. Recent results in time resolved serial femtosecond crystallography at XFELs. Curr. Opin. Struct. Biol. 2020, 65, 193-208. [CrossRef] [PubMed]

26. Kim, K.H.; Kim, J.G.; Nozawa, S.; Sato, T.; Oang, K.Y.; Kim, T.W.; Ki, H.; Jo, J.; Park, S.; Song, C.; et al. Direct observation of bond formation in solution with femtosecond X-ray scattering. Nature 2015, 518, 385-389. [CrossRef]

27. Buzzi, M.; Först, M.; Mankowsky, R.; Cavalleri, A. Probing dynamics in quantum materials with femtosecond X-rays. Nat. Rev. Mater. 2018, 3, 299-311. [CrossRef]

28. Kim, M.; Min, C.-K.; Eom, I. Laser systems for time-resolved experiments at the Pohang Accelerator Laboratory X-ray FreeElectron Laser beamlines. J. Synchrotron Radiat. 2019, 26, 868-873. [CrossRef]

29. Sanchez-Gonzalez, A.; Johnson, A.S.; Fitzpatrick, A.; Hutchison, C.D.M.; Fare, C.; Cordon-Preciado, V.; Dorlhiac, G.; Ferreira, J.L.; Morgan, R.M.; Marangos, J.P.; et al. Coincidence timing of femtosecond optical pulses in an X-ray free electron laser. J. Appl. Phys. 2017, 122, 203105. [CrossRef]

30. Sato, T.; Glownia, J.M.; Ware, M.R.; Chollet, M.; Nelson, S.; Zhu, D. A simple instrument to find spatiotemporal overlap of optical/X-ray light at free-electron lasers. J. Synchrotron Radiat. 2019, 26, 647-652. [CrossRef] [PubMed]

31. Gamaly, E.G. Femtosecond Laser-Matter Interaction: Theory, Experiments and Applications; CRC Press: Boca Raton, FL, USA, 2011.

32. Pham, M.; Yin, P.; Rana, A.; Osher, S.; Miao, J. Generalized proximal smoothing (GPS) for phase retrieval. Opt. Express 2019, 27, 2792-2808. [CrossRef] [PubMed]

33. Redford, S.; Andrä, M.; Barten, R.; Bergamaschi, A.; Brückner, M.; Dinapoli, R.; Fröjdh, E.; Greiffenberg, D.; Lopez-Cuenca, C.; Mezza, D.; et al. First full dynamic range calibration of the JUNGFRAU photon detector. J. Instrum. 2018, 13, C01027. [CrossRef] 